ACTA SCIENTIFIC MICROBIOLOGY (ISSN: 2581-3226)

Volume 2 Issue 10 October 2019

Short Communication

\title{
Monitoring of Microalgae Cultivation Process for Product Potential
}

\author{
Amarjit Singh Sarpal* \\ Instituto Nacional de Metrologia, Qualidade e Tecnologia- INMETRO, Avenida Nossa Senhora das Graças 50, Xerém, Duque de Caxias, RJ, \\ Brazil \\ *Corresponding Author: Amarjit Singh Sarpal, Instituto Nacional de Metrologia, Qualidade e Tecnologia- INMETRO, Avenida Nossa \\ Senhora das Graças 50, Xerém, Duque de Caxias, RJ, Brazil. E-mail: sarpal.as2@gmail.com
}

Received: August 07, 2019; Published: September 05, 2019

DOI: $10.31080 /$ ASMI.2019.02.0367

Algae, a multicellular or unicellular form of living organisms, can be divided into either macro $(>100 \mu \mathrm{m})$ or micro algae $(1-100 \mu \mathrm{m})$ based on its size. Microalgae existence in the ocean is more than 3 billon year old and its diversity falls in the species of prokaryotic cyanobacteria and eukaryotic. There are more than 100000 different types of microalgae species existing in ocean and land water bodies including fresh water (lakes, ponds and rivers). The most potential species studied among these species are only 30,000, which find numerous applications for human beings in terms of biofuels, food, medicines, neutraceuticals, nanotechnology etc. The high yielding species/strains having commercial importance in terms of valuable products such as biofuels, high-protein supplement in human nutrition, aquaculture and nutraceutical with higher bio and lipid productivity include Spirulina, Chlorella, Haematococcus, Dunaliella, Scenedesmus, Botryococcus, Phaeodactylum, Porphyridium, Chaetoceros, Crypthecodinium, Isochrysis, Nannochloris, Nitzschia, Schizochytrium, Tetraselmis, and Skeletonema. Microalgae can convert solar energy into chemical energy possessing highest photosynthetic efficiency and yield of more than 10 times compared to conventional terrestrial agriculture crops. For example, high yielding species such as Spirulina, Botryococcus and Nanochloris can yield up to $50000-130000 \mathrm{l} / \mathrm{ha} / \mathrm{y}$ of oil compared to oil yield of $6000 \mathrm{l} /$ ha/y of palm oil, and $550 \mathrm{l} / \mathrm{ha} / \mathrm{y}$ of soybean oil. Lipid production is modulated by variation of culture parameters such as nitrogen and phosphate concentration, light source and density of photonic flux, metal content, salinity and strain selection in order to enhance lipid productivity with desired fatty acids profile. Altering the biosynthesis pathways through genetic modification has also been adopted as means for lipid yield and quality improvement [1-3].
The biomass from microalgae is a rich source of $30-40 \%$ lipids (Triacyl glycerides; TAG, free fatty acids; FFA), 30-50\% proteins and $10-20 \%$ polysaccharides, starch, food supplements, vitamins, minerals, neutraceuticals, pharmaceuticals, sterols, animal feed and bio-fertilizer [2-7]. The lipids are mainly comprised of triglycerides (TAG), an important component of interest to biodiesel industry. The TG and FFA are composed of both saturated (C14C18) and unsaturated fatty acids (C16-C22) including PUFAs (n-3 omega C18:3, C20:5, C22:6). Biomass of microalgae is an abundant source of vitamins such as vitamins A, B1, B2, C, and E; biotin, folic acid, pantothenic acid, niacin; carotenoids (lipophilic pigments with isoprenoid structures; beta carotene, Astaxanthin, Canthaxanthin, Fucoxanthin, lutein); and minerals potassium, iron, magnesium, calcium, Zn. Mn, Si etc. Microalgae based carotenoids have therapeutic value, including anti-inflammatory and anticancer activities, which are largely attributed to their strong antioxidant effect $[1,2]$. Besides the presence of neutral lipids, TAG (MG, DG, TG) and free fatty acids (FFA), the important classes of polar lipids present include glycero glyco lipids (GL), glycero phospho (GP), sphingo (SP), sterol (ST), prenol (PR), saccharo (SL) and oxylipins (lipoxygenase-derived oxygenated acids of DHA and EPA).These lipids find extensive application in neutraceutical and pharmaceutical industry $[1,8]$.

The commercially exploited microalgae species, Chlorella vulgaris, Spirulina (Arthrospira, plantensis), and Dunaliella (salina and tertiolecta), find extensive application as food supplements such as infant food mix with high nutritional content including essential minerals, which are sold in the form of tablets in Japan 
and other countries. Microalgae frustules, outer shell for housing the cell, are composed of hierarchical architectures, ranging from nanometers to micrometers. Frustules' structure could be used in building highly developed advanced devices such as light harvesting, photonics, semi conductor, molecular separation, sensing, and drug delivery systems [9].

It is quite evident from the ongoing description of microalgae species that chemistry of a biomasses of microalgae is diverse and complex, which are composed of large numbers of novel chemicals possessing unique applications. Thus, an integrated and strategic analytical protocol is required for compositional characterization of a biomass generated by the use of particular microalgae species cultivated in the open or closed photo-bioreactor. As described above, species and nutrients (N, P, K), play an important role in determining the desired composition of a harvested dried biomass. Therefore, a direct, rapid and ease of qualitative or quantitative analyses of a dried biomass or its solvent extract is essentially required by the application of analytical techniques. It should provide rapid results and facilitate the optimization of cultivation parameters such as species/strain selection, nutrient selection, light flux, temperature, choice of photo bio-reactor and polarity of the solvent used for extraction of components for a specific application. Thus, at each step large numbers of dried biomass samples would be cultivated, and required to carry out complete compositional analyses to know the presence, class and nature of most of the components including different classes of lipids. The host of analytical techniques such as chromatographic (GC, GC-MS, HPLC, GPC), molecular spectroscopic (NMR, FTIR, MS, UV and visible) and atomic spectrometry (AAS, ICP-MS, XRF, XRD, TEM, SEM) are being applied for molecular level characterization and recognition of a biomass. Solution state NMR $\left({ }^{1} \mathrm{H},{ }^{13} \mathrm{C}\right)$ techniques have been proved to be authentic, rapid, precise and quantitative, and provide direct analyses without the use of standards [3-8].
Biochemical composition of the algal biomass or its solvent extracts can be altered by selection of proper species/strains and varying cultivation parameters, specifically limiting the $\mathrm{N}$ and $\mathrm{P}$ containing nutrients of the culture. This can significantly enhance the biomass productivity and oil yield, effect the chemical composition and fatty acid profile, and also provide viable options either in favor of biodiesel or value added products. The application of NMR techniques will be demonstrated and highlighted with regards to monitoring of the cultivation process in order to show the effects of change in the nature of microalgae species and nutrients used for cultivation on biomass composition. The Figures 1 and 2 show 500 $\mathrm{MHz}{ }^{1} \mathrm{H}$ NMR spectra of cyclohexane extracts of biomasses generated from two microalgae species namely Chlorela vulgaris (CHL) and Scenedesmus ecornis (SND) cultivated in different nutrient media as explained in the legends of the Figures. On critical interpretation of the spectra, it is clearly indicated that the composition of the biomass is both species and nutrient specific. The essential components of the biomasses such as TAGs and their fatty acid profile comprising of both saturated and unsaturated (C14-C16, C18:n = 0-3, C20:5, C22:6), PUFAs, glycero glycol/phospho lipids, sterols, carotenoids and oxylipins could conveniently be characterized from a single spectrum. The analyses have been carried out on solvent extracts of biomasses as such without resorting to the route of trans-esterification as usually adopted by chromatographic techniques. Besides the component analyses, the quantitative analyses including total lipid content of biomass has also been achieved by the developed methods based on the integral area measurements of the respective components as described in the references [3-7]. The PUFA contents of different oil extracts of different biomasses generated in fresh and industrial waste water estimated by method based ion ${ }^{1} \mathrm{H}$ NMR is shown in Figure 3 [7]. Thus single technique analytical approach is strategically rapid and convenient to predict real performance of algal strains for biomass growth and productivity mapping.

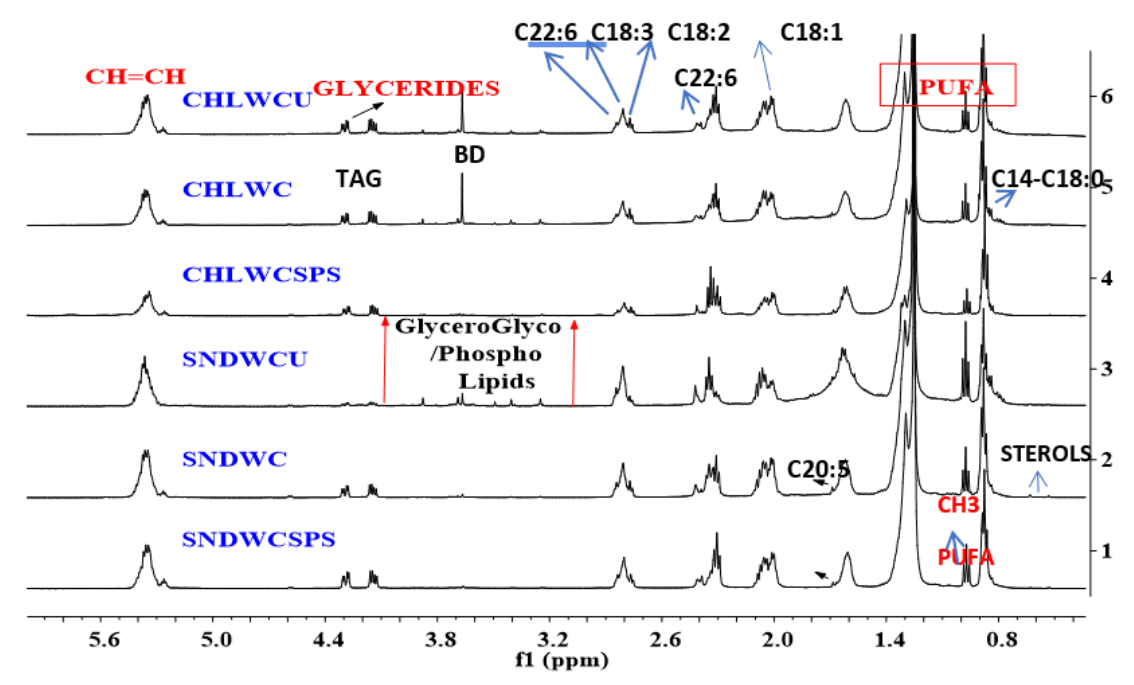

Figure 1: $500 \mathrm{MHz}{ }^{1} \mathrm{H}$ NMR part spectra of cyclohexane extracts (algal oils) of biomasses generated by microalgae species Chlorella

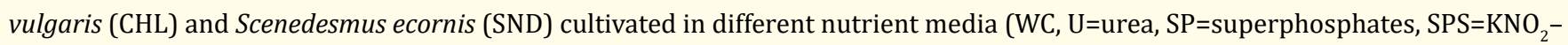
superphosphates, $\mathrm{S}=\mathrm{KNO}_{2}$. 


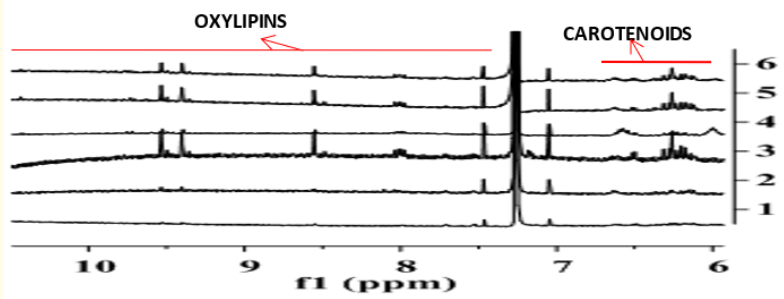

Figure 2: $500 \mathrm{MHz}^{1} \mathrm{H}$ NMR part spectra (Figure 1) of cyclohexane extracts (algal oils) of biomasses generated by microalgae species Chlorella vulgaris (CHL) and Scenedesmus ecornis (SND) cultivated in different nutrient media as described in the legend of Figure 1.

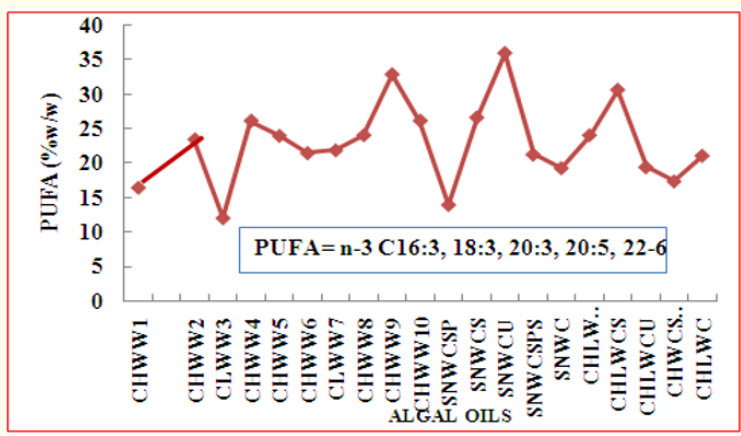

Figure 3: PUFAs content of different algal oils extracted from various biomasses generated by microalgae species Chlorella vulgaris (CHL) and Scenedesmus ecornis (SN) cultivated in different nutrient media (WC, $\mathrm{U}=$ urea, $\mathrm{SP}=$ superphosphates, SPS $=\mathrm{KNO}_{2}$-superphosphates, $\mathrm{S}=\mathrm{KNO}_{2}$ ), WW1-10= cultivation in industrial waste water [7].

The components characterization of microalgae extracts for different lipid classes and metabolites have been carried out by traveling wave ion mobility mass spectrometry (TWIM-MS). Two different analysis strategies have been reported namely direct infusion electro spray ionization (ESI) with no previous chromatographic separation (DI-ESI-TWIM-MS), and for metabolites and lipids using an untargeted high-throughput method that employs ultra high-performance liquid chromatography (UHPLC) using data-independent analysis (DIA)-MSE (UHPLC-HDMSE).With the UHPLC-HDMSE approach, 1251 different compounds were identified across 16 samples with 210 classified as lipids that include different classes of lipids such as triacyl glycerides (TAG), glycerol lipids (GL), glycerophosphate lipids (GP), Sphingosine lipids (SP), sterol lipids (ST), prenol lipids (PR), and saccharo lipids (SL) [8].
Thus methods based on NMR spectroscopic techniques facilitate rapid screening of algal species or strains for cultivation of biomass with the desired lipid content, quality, and potential for biodiesel as well as value-added co-products in view of the economics of the overall cost of generation of the biomass. Mass spectrometric techniques are useful for providing detailed information regarding profiling of different classes of lipids.

\section{Bibliography}

1. Khan MI., et al. "The promising future of microalgae: current status, challenges, and optimization of a sustainable and renewable industry for biofuels, feed, and other products". Microbial Cell Factories 17.1 (2018): 36.

2. Sathasivam R., et al. "Microalgae metabolites: A rich source for food and medicine". Saudi Journal of Biological Sciences 26 (2019): 709-722.

3. Sarpal AS., et al. "Determination of lipid content of oleaginous microalgae biomass by NMR spectroscopic and GC-MS techniques". Analytical and Bioanalytical Chemistry 407.13 (2015): 3799-3816.

4. Sarpal AS., et al. "NMR techniques for determination of lipid content in microalgal biomass and their use in monitoring the cultivation with biodiesel potential". Applied Microbiology and Biotechnology 100.5 (2015): 2471-2485.

5. Sarpal AS., et al. "Investigation of Biodiesel Potential of Biomasses of Microalgaes Chlorella, Spirulina and Tetraselmis by NMR and GC-MS Techniques". Journal of Biotechnology and Biomaterials 6 (2016): 220.

6. Sarpal., et al. "Biodiesel and product potential of microalgae Dunaliella Salina by NMR Techniques". Advances in Biotechnology and Microbiology 11.3 (2018): 555812.

7. Sarpal AS., et al. "Biodiesel and Polyunsaturated fatty acid (PUFA) potential of Microalgae biomass- A Short Review". RDMS 10.4 (2019): 000744.

8. Fasciotti M., et al. "Investigating the Potential of Ion Mobility-Mass Spectrometry for Microalgae Biomass Characterization". Analytical Chemistry 114.9 (2019): 266-9276.

9. Jamali AK., et al. "Applications of Diatoms as Potential Microalgae in Nano biotechnology". BioImpacts 2.2 (2012): 83-89.

\section{Volume 2 Issue 10 October 2019}

(C) All rights are reserved by Amarjit Singh Sarpal. 